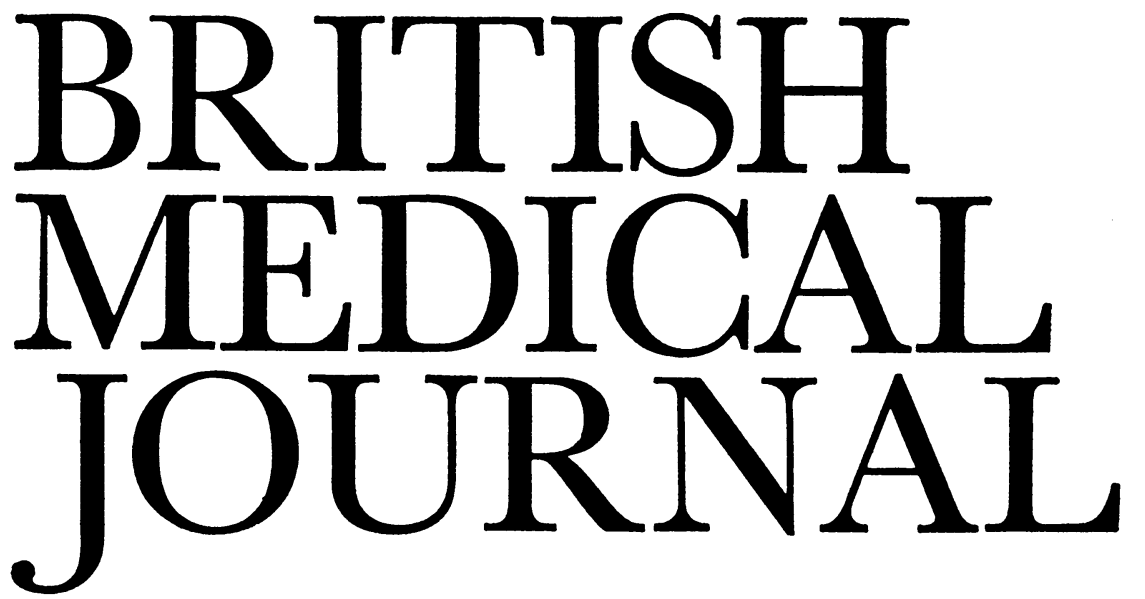

LONDON SATURDAY 24 JULY 1971

\title{
Human Prolactin
}

Although prolactin has long been accepted as a distinct pituitary hormone in most animals, it is only recently that sufficient evidence has accumulated to confirm its individual existence in man. ${ }^{1}$ It shows many similarities in structure and function to growth hormone and to the lactogenic hormone derived from the placenta-placental lactogen.

Secretion of prolactin, like that of melanocyte-stimulating hormone but not the other hormones of the anterior lobe of the pituary, is inhibited by a hypothalamic factor termed prolactin-release-inhibiting hormone. ${ }^{2}$ This substance, which by analogy with the hypothalamic releasing factors isolated so far, is likely to be a short-chain polypeptide, is synthesized in the hypothalamus, stored in the median eminence, and transported to the anterior lobe in the portal venous system, which passes down the pituitary stalk. There is some evidence that it is synthesized in the same area of the hypothalamus as a factor affecting the release of luteinizing hormone, which might explain the frequent association of galactorrhoea and secondary amenorrhoea. Galactorrhoea in the male is apt to be associated with gynaecomastia.

Many causes of inappropriate lactation probably act by antagonizing the prolactin-release-inhibiting hormone. They include drugs known to affect the hypothalamus such as reserpine and chlorpromazine; diseases affecting the hypothalamus, such as tumours and granulomas; and section of the pituitary stalk either surgically or by a tumour or trauma. In addition certain pituitary tumours have been shown to contain prolactin. ${ }^{3}$

Evidence for the independent existence of prolactin comes from many sources, clinical, biochemical, and histological. Clinically it has long been known that sexual ateliotic dwarfs, who have an isolated defect in the formation of growth hormone, can initiate and maintain lactation despite the absence of the growth hormone. More recently a rise in serum prolactin has been found in such a dwarf during pregnancy, ${ }^{4}$ and it showed a further dramatic rise in response to suckling. Studies reported by Dr. I. A. Forsyth and her colleagues in this week's B.M.F. at page 225 further confirm the existence of prolactin. Using a bioassay depending on the lactogenic response of rabbit mammary tissue in organ culture, ${ }^{5}$ they have detected raised levels of prolactin, distinct from growth hormone, in the plasma of patients of both sexes with inappropriate lactation, with or without pituitary tumours. Prolactin was absent from the plasma of patients with gynaecomastia without galactorrhoea. Levels of prolactin were not depressed by administration of glucose to patients with galactorrhoea associated with pituitary tumours, whereas in those without enlargement of the pituitary fossa glucose did depress the level of prolactin. If further studies confirm these observations, clinicians will have a useful test for separating among patients with galactorrhoea those with and those without organic pituitary lesions.

Treatment of galactorrhoea has always presented problems, because only a minority of patients improve spontaneously or respond to clomiphene. Dr. P. M. Lutterbeck and his colleagues, also reporting in this week's B.M.F. (page 228), describe the use of an ergot alkaloid for the treatment of three patients with non-puerperal galactorrhoea, one of whom had an enlarged pituitary fossa. The drug reduced the secretion of milk in all three patients but had to be discontinued in one because of side effects, which included dizziness and nausea.

Histological studies in man have also provided evidence for the existence of prolactin. As in animals, certain acidophil cells in the anterior pituitary stain with specific dyes such as azocarmine or erythrosin. These cells are rare in the pituitary under normal conditions but show a dramatic increase during pregnancy and lactation. ${ }^{6}$ They can easily be distinguished from other smaller acidophil cells secreting growth hormone, which stain specifically with orange $G$ and contain smaller secretory granules. Selective immunofluorescent stains confirm the secretion of prolactin and growth hormone from separate types of acidophil cells. ${ }^{7}$

Actual measurements of prolactin seem to correlate well with predictions based on clinical observations. What is now needed is a reliable and simple method of measuring the hormone, since existing bioassays are cumbersome, time-consuming, and difficult to quantitate, and immunoassays are at present based on cross-reactions with animal prolactin. Purification of human prolactin from tissue or organ cultures should soon allow the development of specific and sensitive immunoassays which can be applied in clinical practice.

1 Sherwood, L. M., New England Fournal of Medicine, 1971, 284, 774.

2 Schally, A. V., et al., Recent Progress in Hormone Research, 1968, 24, 497. 3 Peake, G. T., et al., fournal of Clinical Endocrinology and Metabolism, 1969 , $29,1383$. 
4 Hwang, P., Guyda, H., Friesen, H., and Tyson, J., Abstracts of the Endocrine Society, 24 June 1971, San Francisco, p. A-43.

${ }^{5}$ Forsyth, I. A., fournal of Endocrinology, 1969, 46, 4.

6 Goluboff, L. G., and Ezrin, C., Fournal of Clinical Endocrinology and Metabolism, 1969, 29, 1533

' Herbert, D. C., and Hayashida, T., Science, 1970, 169, 378.

\section{A Kidney from the Family}

Since kidney grafting was attempted therapeutically more than 15 years ago it has been clear that the overall results of transplants donated from close blood relatives have been considerably better than those from unrelated donors, whether live volunteers or cadavers. When the donor is an identical twin and there can be no immunological rejection, the two-year functional survival rate of kidneys is $87 \%$, and the longest a patient has survived is now 15 years. In donors who are close blood relatives but not identical twins the two-year survival rate for kidneys is $75 \%$ and the longest survival period 12 years. In contrast, for kidneys from unrelated cadaver donors the two-year survival rate it $41 \%$ and the longest a functioning kidney has survived is now seven years. The main reason why family donors are better than unrelated is because the transplantation antigens are inherited in a similar way to red-cell antigens, and therefore the chances of some degree of matching between donor and recipient are higher than in a random unrelated population.

The results, however, are better than can simply be explained on inheritance of the major transplantation antigens. They could be due to a cumulative effect of minor transplantation antigens not at present recognized as important or to some immunological interaction between parent and child which tends to lessen the danger of rejection, since it is in this group particularly that the results are better than would be expected. An important surgical advantage with a live donor is that the ischaemic damage suffered by the kidney is likely to be minimal, and the operation can be carefully planned without any element of emergency or rush. Probably many transplants from cadaver donors have failed from non-immunological causes-in particular ischaemic damage and also surgical errors resulting from the circumstances in which cadavic renal transplantation is practised.

Despite all these factors in favour of live donors there has been much concern, especially among surgeons in the United Kingdom, about using their kidneys, since the preoperative assessment and the nephrectomy itself have small but definite risks for the healthy donor and always entail discomfort and a certain degree of pain. Even more important has been the fear that the doctor would unwillingly let loose much unhappiness and distress on the family forced to decide which member should or should not be considered as a donor. Most surgeons in the United Kingdom are prepared to perform transplants from living donors only if the donor is a very close relative and persuades the doctor that he will feel deprived if denied this opportunity to help his loved one. Thus the doctor tends to act as "Devil's advocate," persuading the donor against offering his kidney rather than encouraging him.

In several centres in the United States the opposite view has been taken. This is that, since a live donor gives the best chances of success, the whole family should, as a duty, consider who among them should be medically assessed as a potential donor. In fact, acceptance of recipients in some transplant centres is dependent on there being a suitable family donor available, otherwise, owing to shortage of beds and staff, transplantation cannot be performed. This is widely understood in the general population, and if somebody is afflicted with renal disease his family immediately rallies round to see who could be a donor. Members of the family may even present themselves as potential donors to the doctor before the question of transplantation has been seriously raised. This is certainly not a familiar pattern in the United Kingdom, where it is unusual for the patient's family to volunteer to give a kidney.

An important study recently undertaken by R. G. Simmons and colleagues ${ }^{1}$ from the University of Minnesota has shown that the search for a kidney transplant donor in a family can cause considerable stress. Tension was noted in 21 of 79 cases studied, and it was concluded that previously the nature of this crisis had been minimized. Insufficient attention had been paid to the family as an interacting unit or to those members of the family who could have donated but did not.

Usually when children were the recipients they did not generate the same crisis of decision-making as did adult recipients. Parental sacrifice for a child seems to be accepted. The decision to donate is not usually difficult for a parent; in only 5 of 21 cases was family tension noted.

For an adult recipient a sibling is the best donor genetically. But in 13 of 19 cases considerable family tension involved the potential sibling donor. There was ambivalence on the part of donors and conflict between the rest of the family. In eight cases all siblings who were asked to donate either refused directly or avoided contact with the transplant service and the patient. Criticism was expressed by some members of families for the great pressure exerted on individuals to be donors. In three cases the spouse of the recipient made tremendous efforts to round up potential donors within the family. Recipients have expressed bitterness towards the family. However, when conflicts have been followed by a successful transplant, they may be resolved. In several instances the donor's spouse has actively opposed donation.

The results of this study should serve as a caution to the overenthusiastic acceptance of a donor within the family. It is desirable that there should be pressure from the would-be donor to convince the doctor, who in turn must point out all the possible dangers.

' Simmons, R. G., Hickey, K., Kjellstrand, C. M., and Simmons, R. L., Fournal of the American Medical Association, 1971, 215, 909.

\section{Psychogeriatric Care}

In future psychiatric care is to be integrated into the body of general hospital medicine. ${ }^{1}$ Elderly patients with organic brain disease are to become the province of the geriatrician. ${ }^{2}$ Yet in the face of a general decline in the psychiatric inpatient population the proportion of elderly admissions to psychiatric hospitals is on the increase. ${ }^{3}$ Some geriatric physicians have expressed concern at the increased burden of psychiatric disorder that they already have to bear and hope that the advent of the psychogeriatric unit may help to reduce it. ${ }^{4}$

The collective views of psychiatrists working in psychiatric hospitals have recently been expressed in a report 